\title{
QUALIDADE DE VIDA DE ADULTOS COM DEFICIÊNCIA VISUAL NA GRANDE FLORIANÓPOLIS, SC
}

\author{
Roger Lima Scherer \\ Universidade Federal de Santa Catarina, Florianópolis, Santa Catarina, Brasil. \\ Luciano Lazzaris Fernandes \\ Universidade Federal de Santa Catarina, Florianópolis, Santa Catarina, Brasil. \\ Markus Viniciusa Nahas \\ Universidade Federal de Santa Catarina, Florianópolis, Santa Catarina, Brasil.
}

\begin{abstract}
Resumo
O objetivo deste estudo foi avaliar qualidade de vida (q.v.) em adultos com deficiência visual (d.v.). Foram entrevistadas 168 pessoas (18 a 59 anos). Para a coleta de dados foi utilizada uma entrevista semiestruturada. A percepção de q.v. apresentou uma tendência quando analisada sob o período de incidência da deficiência. Quanto mais tarde as pessoas adquiriram a d.v., menor foi sua percepção positiva com relação à q.v. As mulheres jovens, sem companheiros, com nove anos ou mais de estudo, que trabalham, foram as que apresentaram melhor percepção de q.v. entre os adultos com d.v. Deve-se pensar em estratégias de promoção para saúde, com viés de melhorias na q.v. dos adultos que apresentaram uma prevalência maior para a percepção negativa.
\end{abstract}

Palavras chave: Qualidade de vida. Deficientes visuais. Condições de vida. Estilo de vida.

\section{Introdução}

Desde a década de 1960 a expressão qualidade de vida tem recebido atenção crescente, passando de retórica de políticos em campanha para tema de pesquisa e referência para formulação de políticas públicas.

Por ser um tema relevante e conceitualmente multidisciplinar, a qualidade de vida das pessoas e de nações tem sido objeto de estudo de inúmeras áreas, incluindo a Sociologia, a Educação, a Saúde Pública e a Educação Física (GILL; FEINSTEIN, 1994; FLECK et al., 1999; NAHAS, 2010).

Seidl e Zannon (2004) enfatizaram que, na área da saúde, o interesse pelo conceito de qualidade de vida é relativamente recente e decorre, em parte, dos novos paradigmas que têm influenciado as políticas e as práticas do setor nas últimas décadas.

Não há um consenso sobre a definição de qualidade de vida. Entretanto, admite-se que há múltiplos fatores determinantes dessa condição humana, assim como a influência de diversos domínios, tais como: o meio ambiente, recursos econômicos, relacionamentos, atenção à saúde, condições de trabalho e oportunidades de lazer. Dessa forma, diversos fatores podem estar associados ao constructo qualidade de vida, sendo extremamente complexa a tarefa de medi-la. Dantas, Sawada e Malerbo (2003) corroboraram a ideia de 
que a qualidade de vida apresenta inúmeros significados, pois é uma noção eminentemente humana, que reflete conhecimentos, experiências e valores de indivíduos e coletividades. Esses significados expressam o momento histórico e as condições de vida (aspectos ambientais, econômicos e socioculturais).

Nahas (2013, p. 15), numa perspectiva holística, afirma que a qualidade de vida pode ser entendida como a "percepção de bem-estar resultante de um conjunto de parâmetros individuais e socioambientais, modificáveis ou não, que caracterizam as condições em que vive o ser humano". Nessa perspectiva, está associada com o estado de saúde, longevidade, salário e satisfação no trabalho, opções de lazer, relações familiares, disposição, prazer e até espiritualidade.

Diversos grupos populacionais têm sido estudados e inúmeras medidas de qualidade de vida estão disponíveis na literatura especializada (GILL; FEINSTEIN, 1994; FLECK, 2000). Quer no enfoque holístico anteriormente mencionado ou na perspectiva da saúde ou do desenvolvimento humano (como índice populacional, a exemplo do IDH da ONU), investigar a qualidade de vida vem sendo cada vez mais importante para identificar necessidades e propor meios de melhorar a condição de vida de indivíduos e populações.

Um segmento populacional menos investigado em questões de qualidade de vida (particularmente no Brasil) tem sido o das pessoas com deficiência (BROWN, 1997). Considerando que a qualidade da visão também é um determinante importante da qualidade de vida de todos os indivíduos, não é difícil entender que sua limitação traz dificuldades em ações simples da vida diária, como: vestir-se, alimentar-se ou banhar-se, até na comunicação simples com outras pessoas ou interação com o ambiente (STELMACK, 2001). A perda de visão (parcial ou total) pode provocar alterações significativas na vida das pessoas, exigindo grandes adaptações para uma vida produtiva e feliz na sociedade, como sugerem Bittencourt e Hoehne (2006).

Campos e Neto $(2008$, p. 235) relataram que há instrumentos específicos capazes de "avaliar, de forma individual e específica, determinados aspectos da qualidade de vida, proporcionando maior capacidade de detecção de melhora ou piora do aspecto em estudo". Entretanto não foi encontrado na literatura um instrumento para mensurar a qualidade de vida, especifico para adultos com deficiência visual. Também não se encontrou informação a esse respeito na população de pessoas com deficiência visual no Estado de Santa Catarina. Assim, o objetivo deste estudo exploratório foi avaliar a qualidade de vida em adultos com deficiência visual residentes na Grande Florianópolis.

\section{Método}

A pesquisa foi realizada com pessoas com deficiência visual, diagnosticadas de acordo com critérios da Organização Mundial da Saúde. Essas pessoas, com idades de 18 a 59 anos, estavam cadastradas na Associação Catarinense para Integração dos Cegos (ACIC) da Grande Florianópolis. Foram incluídos apenas os adultos que possuíam condições de responder à entrevista sem auxílio de terceiros.

A ACIC possui reconhecimento nacional e internacional pelo exemplar trabalho nas áreas de reabilitação, profissionalização e reintegração das pessoas com deficiência visual, atendendo muitas pessoas com deficiência visual do Estado de Santa Catarina. 
Pretendia-se fazer o levantamento com todos os associados da entidade (ACIC) que atendiam aos critérios de inclusão no estudo, realizando-se, dessa forma, um censo sobre a percepção da qualidade de vida dos adultos com deficiência visual da Grande Florianópolis. Estavam cadastrados na entidade 776 pessoas, sendo elegíveis para o estudo apenas 321 associados na faixa etária desejada (18 a 59 anos). A amostra final foi de 168 adultos, considerando que 11 indivíduos se recusaram a participar do estudo, 87 não foram encontrados e 55 não participaram por dificuldades de contato para realizar a coleta, após várias tentativas.

Para a coleta das informações foi utilizado um roteiro de entrevista, elaborado a partir do modelo proposto por Nahas (2010), adaptado para pessoas com deficiência visual com base na literatura especializada e, principalmente, nos indicadores evidenciados em um grupo focal com adultos com deficiência visual.

$O$ roteiro de entrevista utilizado neste estudo foi previamente testado com relação à validade de face e conteúdo, clareza (pré-teste) e reprodutibilidade (estudo piloto com aplicações sucessivas). O processo de construção e validação está descrito na dissertação de Scherer (2012) e incluiu a análise de profissionais experientes na área de educação especial. Em todas as etapas seguiram-se os preceitos éticos, informando sobre o sigilo no tratamento das informações utilizadas, e os entrevistados concordaram em participar, mediante a assinatura do TCLE (Termo de Consentimento Livre e Esclarecido).

A concepção de qualidade de vida operacionalizada para este estudo (Figura 1) foi definida em um grupo focal, com base no modelo de qualidade de vida proposto por $\mathrm{Na}$ has (2010).

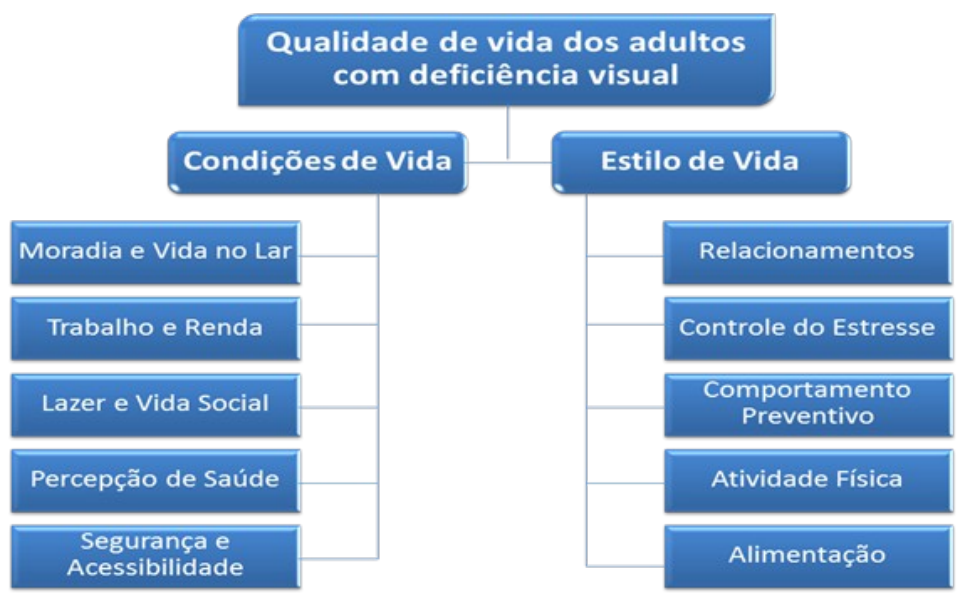

Figura 1 - Modelo proposto para definição e avaliação da qualidade de vida dos adultos com deficiência visual (SCHERER, 2012).

Protocolo de pontuação para avaliação da qualidade de vida dos adultos com deficiência visual 


\section{Dimensão 1 - Condições de vida}

A dimensão condições de vida foi analisada por meio de 16 questões em cinco componentes: moradia e vida no lar (3), trabalho e renda (3), lazer e vida social (3), percepção de saúde (3), segurança e acessibilidade (4). Em todas as questões utilizou-se uma escala de 0 a 3 ( 0 e 1 - percepção negativa; 2 e 3 - percepção positiva).

O indivíduo que não trabalhava recebeu uma percepção negativa no componente trabalho e renda.

O componente segurança e acessibilidade possui quatro variáveis, sendo duas relativas à segurança (questões 49 e 50), uma no lar e outra nas ruas, durante o deslocamento. As outras duas são referentes a acessibilidades (questões 51 e 52).

As questões referentes à percepção de acessibilidade atitudinal e arquitetônica possuem cinco ambientes diferentes (lar, áreas de lazer, áreas comerciais, trabalho e deslocamento). Cada ambiente apresenta um escore crescente, de acordo com sua resposta (0 a 3). Em cada questão, o somatório dos escores dos ambientes resultou na percepção positiva ( $\geq 8)$ ou negativa da acessibilidade atitudinal e arquitetônica.

Dessa forma, a análise no componente segurança e acessibilidade realizou-se com quatro variáveis: segurança no lar e segurança na rua, com escores 0 e 1 - percepção negativa; 2 e 3 - percepção positiva; acessibilidade arquitetônica e acessibilidade atitudinal, com 0 - negativa e 1 - positiva. No componente segurança e acessibilidade, o indivíduo teve uma percepção positiva quando o sujeito obteve três ou mais variáveis positivas.

Os componentes que possuíam percepção negativa obtiveram escore 0 (zero), e aqueles com percepção positiva, escore 1 (um). Dessa forma, nas condições de vida o indivíduo teve um somatório entre 0 e 5, considerando-se 0 e 1 - percepção negativa; 2 e 3 - percepção regular; 4 e 5 - percepção positiva.

\section{Dimensão 2 - Estilo de vida}

A dimensão estilo de vida foi analisada por meio de 15 questões em cinco componentes: relacionamentos, controle do estresse, comportamento preventivo, atividade física e alimentação.

No componente relacionamentos há três variáveis com escala de Likert (0 e 1 negativo; 2 e 3 - positivo), sendo que ele foi considerado positivo se duas variáveis apresentarem comportamento positivo (escores dois e três).

No componente controle do estresse há duas variáveis com escala de Likert ( 0 e 1 - negativo; 2 e 3 - positivo) e uma variável sobre o tempo dedicado ao sono, sendo que foi considerado positivo, nessa variável, se o indivíduo dedicar 7 horas ou mais para seu sono. Esse componente foi considerado positivo se duas variáveis apresentarem comportamento positivo (escores dois e três).

No componente comportamento preventivo as variáveis foram consideradas como comportamento positivo ou negativo da seguinte forma:

Questão 35 - Faz exames de rotina? (não - negativo / sim - positivo).

Questão 36 - Fuma? (sim - negativo / não - positivo).

Questão 37 - Bebe? (sim " $\geq 8$ para mulheres e $\geq 15$ para homens" - negativo / sim “ $\leq 7$ para mulheres e $\leq 14$ para homens" ou não - positivo). 
Esse componente foi considerado positivo quando duas variáveis apresentaram comportamento positivo (escores dois e três).

No componente atividade física, o indivíduo recebeu escore 0 a 3, de acordo com o nível de atividade física (frequência semanal e duração de prática), baseado nas recomendações da WHO (2010).

0 - Inativo: (não realiza atividades físicas / até 74 min. praticando apenas uma vez por semana).

1 - Cumpre pouco as recomendações: (até 74 min. de duração e frequência acima de duas vezes por semana / entre 75 e 149 min. praticados em até duas vezes por semana).

2 - Cumpre parcialmente as recomendações: (entre 75 e 149 min. praticados acima de três vezes por semana / acima de $150 \mathrm{~min}$. praticados em até duas vezes por semana).

3 - Cumpre as recomendações: (acima de 150 min. praticados acima de três vezes por semana).

Tal componente foi considerado positivo quando o indivíduo cumpriu parcialmente as recomendações ou cumpriu as recomendações.

No componente alimentação, as variáveis foram consideradas como comportamento positivo ou negativo, da seguinte forma: sitivo).

Questão 43 - Quantos dias por semana você ingere frutas? $(<5$ - negativo / $\geq 5$ po-

Questão 44 - Quantos dias por semana você ingere alimentos gordurosos? $(\geq 5$ negativo / $<5$ positivo).

Questão 45 - Quantos dias por semana faz de 4 a 5 refeições? $(<5$ - negativo / $\geq 5$ positivo).

Esse componente foi considerado positivo quando duas variáveis apresentaram comportamento positivo (escores dois e três).

$\mathrm{O}$ estilo de vida do indivíduo foi avaliado de acordo com um somatório entre 0 e 5, considerando-se: 0 e 1 - comportamento negativo; 2 e 3 - comportamento regular; 4 e 5 - comportamento positivo.

Cada componente nas duas dimensões foi dicotomizado em negativo (recebendo escore 0 ) e positivo (recebendo escore 1). As condições de vida e o estilo de vida do indivíduo foram avaliados de acordo com um somatório dos respectivos componentes, podendo variar de 0 a 5.

\section{Percepção de qualidade de vida}

A percepção de qualidade de vida foi determinada pelo somatório dos escores positivos das duas dimensões do instrumento (condições de vida e estilo de vida dos adultos com deficiência visual). Assim, os indivíduos receberam uma pontuação entre 0 e 10, considerando-se percepção negativa $\leq 4$; percepção intermediária, entre 5 e 7 ; e percepção positiva de 8 a 10 pontos, conforme Figura 2. 


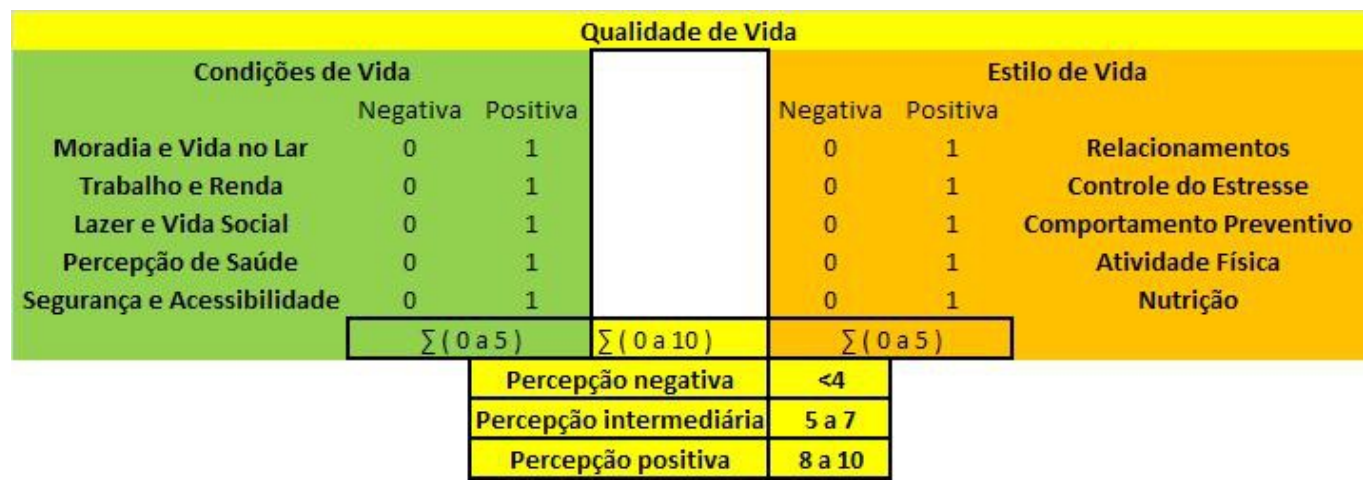

Figura 2. Escores referentes à percepção de qualidade de vida dos adultos com deficiência visual (SCHERER, 2012).

Para verificar a associação entre a variável desfecho (qualidade de vida), em relação aos indicadores sociodemográficos (sexo, idade, escolaridade, trabalho e estado civil), indicador de saúde (IMC) e indicadores visuais (resquício visual e período de incidência) foi realizada uma regressão logística multinomial.

O projeto de pesquisa foi aprovado pelo Comitê de Ética em Pesquisa com Seres Humanos da Universidade Federal de Santa Catarina, sob o no 1930/2011. Todos os indivíduos incluídos na amostra concordaram em participar do estudo após ouvirem a leitura do TCLE, que incluía informações a respeito dos objetivos da pesquisa e a garantia de anonimato.

\section{Resultados}

Foram incluídos na pesquisa adultos de 18 a 59 anos de idade, com média igual a 37,55 anos $(\mathrm{DP}=11,55)$. Não houve diferença entre os gêneros quanto à idade. Entre os participantes $(\mathrm{n}=168), 51,2 \%$ eram homens, 63,7\% residiam em Florianópolis e 56,5 tinham o curso médio ou superior. Essas e outras informações dos sujeitos estão na Tabela 1.

Tabela 1. Características sociodemográficas, de acordo com o sexo, dos adultos com deficiência visual da Grande Florianópolis.

\begin{tabular}{|c|c|c|c|c|c|}
\hline \multirow[b]{2}{*}{ Faixa etária } & \multicolumn{2}{|c|}{ Masculino } & \multicolumn{2}{|c|}{ Feminino } & \multirow{2}{*}{$\begin{array}{c}\text { Total } \\
\text { n (\%) }\end{array}$} \\
\hline & $\mathbf{n}$ & $\%$ & $\mathbf{n}$ & $\%$ & \\
\hline Adultos Jovens (18 a 39 anos) & 50 & 58,1 & 46 & 56,1 & $96(57,1 \%)$ \\
\hline Meia-idade (40 a 59 anos) & 36 & 41,9 & 36 & 43,9 & $72(42,9 \%)$ \\
\hline Total & 86 & 100 & 82 & 100 & $168(100 \%)$ \\
\hline Município & $\mathbf{n}$ & $\%$ & $\mathbf{n}$ & $\%$ & n (\%) \\
\hline Florianópolis & 52 & 60,5 & 55 & 67,1 & $107(63,7 \%)$ \\
\hline São José & 17 & 19,8 & 13 & 15,9 & $30(17,9 \%)$ \\
\hline Palhoça & 9 & 10,5 & 6 & 7,3 & $15(8,9 \%)$ \\
\hline Tempo que reside & $\mathbf{n}$ & $\%$ & $\mathbf{n}$ & $\%$ & n (\%) \\
\hline Até 11 meses & 8 & 9,3 & 12 & 14,6 & $20(11,9 \%)$ \\
\hline Entre 1 e 5 anos & 18 & 20,9 & 19 & 23,2 & $37(22,0 \%)$ \\
\hline Entre 6 e 10 anos & 21 & 24,4 & 13 & 15,9 & $34(20,2 \%)$ \\
\hline
\end{tabular}

Pensar a Prática, Goiânia, v. 17, n. 4, out./dez. 2014 


\begin{tabular}{lccccc}
\hline Acima de 11 anos & 39 & 45,3 & 38 & 46,3 & $77(45,8 \%)$ \\
\hline Nível escolar & $\mathbf{n}$ & $\mathbf{\%}$ & $\mathbf{n}$ & $\mathbf{\%}$ & $\mathbf{n}(\mathbf{\%})$ \\
$\leq$ Fundamental incompleto & 20 & 23,3 & 18 & 22,0 & $38(22,7 \%)$ \\
Fundamental completo & 16 & 18,6 & 19 & 23,2 & $35(20,8 \%)$ \\
Ensino médio & 32 & 37,2 & 26 & 31,7 & $58(34,5 \%)$ \\
Ensino superior ou Pós-Graduação & 18 & 20,9 & 19 & 23,2 & $37(22,0 \%)$ \\
\hline Estado civil & $\mathbf{n}$ & $\mathbf{\%}$ & $\mathbf{n}$ & $\mathbf{\%}$ & $\mathbf{n}(\mathbf{\%})$ \\
Solteiro & 45 & 52,3 & 32 & 39,0 & $77(45,9 \%)$ \\
Casado ou vivendo como casado & 35 & 40,7 & 41 & 50,0 & $76(45,2 \%)$ \\
Separado ou viúvo & 6 & 7,0 & 9 & 5,4 & $15(8,9 \%)$ \\
\hline Local de moradia & $\mathbf{n}$ & $\mathbf{\%}$ & $\mathbf{n}$ & $\mathbf{\%}$ & $\mathbf{n}(\mathbf{\%})$ \\
Casa & 59 & 68,6 & 59 & 72,0 & $118(70,2 \%)$ \\
Apartamento & 27 & 31,4 & 23 & 28,0 & $50(29,8 \%)$ \\
\hline Propriedade do imóvel & $\mathbf{n}$ & $\mathbf{\%}$ & $\mathbf{n}$ & $\mathbf{\%}$ & $\mathbf{n}(\mathbf{\%})$ \\
Próprio & 56 & 65,1 & 55 & 67,1 & $111(66,1 \%)$ \\
Alugado & 30 & 34,9 & 27 & 32,9 & $57(33,9 \%)$ \\
\hline Com quem mora & $\mathbf{n}$ & $\mathbf{\%}$ & $\mathbf{n}$ & $\mathbf{\%}$ & $\mathbf{n}(\mathbf{\%})$ \\
Sozinho & 9 & 10,5 & 8 & 9,8 & $17(10,1 \%)$ \\
Familiares & 66 & 76,7 & 70 & 85,4 & $136(81,0 \%)$ \\
Outras pessoas & 11 & 12,8 & 4 & 4,9 & $15(8,9 \%)$ \\
\hline
\end{tabular}

Pouco mais da metade $(54,2 \%)$ dos adultos com deficiência visual residentes na Grande Florianópolis trabalhavam de forma remunerada, com proporções semelhantes entre homens e mulheres. Entre as ocupações exercidas, as mais frequentes eram: massoterapia $(8,3 \%)$, serviços administrativos $(5,9 \%)$, reciclagem de materiais $(5,9 \%)$ e telefonista $(5,9 \%)$. Serviços pedagógicos e/ou de docência foram referidos por $10,1 \%$ dos respondentes, havendo também no grupo um psicólogo, autônomo (vendas), artesão, radialista e assistente social, entre outras ocupações.

Dentre aqueles que não trabalhavam, as razões mais citadas foram: receber aposentadoria $(43,6 \%)$, algum outro tipo de auxílio financeiro $(14,1 \%)$, ou o fato de estar estudando. Apesar de estarem amparados pelas leis e haver uma abertura razoável para o mercado de trabalho em Santa Catarina, algumas pessoas ainda relataram não estar trabalhando em decorrência da deficiência $(5,1 \%)$.

\section{Percepção das condições de vida}

Os adultos com deficiência visual residentes na Grande Florianópolis apresentaram escore médio de $3,29(\mathrm{DP}=1,21)$, com mínimo de 0 e máximo de 5 pontos para as condições de vida. Escores 4 ou 5 correspondiam à percepção positiva na dimensão condições de vida.

Em geral, $46 \%$ dos adultos com deficiência visual apresentaram percepção positiva para as condições de vida, sendo que os adultos cegos relataram percepções positivas nesse domínio com mais frequência do que aqueles com baixa visão $(51,9 \%$ e $41,8 \%$ respectivamente). A Tabela 2 mostra os percentuais de sujeitos com percepção positiva nos diversos componentes da dimensão condições de vida.

Quando analisados de acordo com o sexo, os homens apresentaram percepção positiva das condições de vida com mais frequência do que as mulheres $(50,0 \%$ e $42,7 \%$ 
respectivamente). Pessoas cegas do gênero masculino foram os que apresentaram a maior prevalência de percepção positiva das condições de vida.

Tabela 2. Percentuais de percepção positiva das condições de vida ${ }^{1}$ - Adultos com deficiência visual da Grande Florianópolis, por grau de deficiência e gênero.

\begin{tabular}{|c|c|c|c|c|c|}
\hline \multirow{2}{*}{ Componentes } & \multicolumn{2}{|c|}{ Cego } & \multicolumn{2}{|c|}{ Baixa Visão } & \multirow{2}{*}{ Geral } \\
\hline & Mulheres & Homens & Mulheres & Homens & \\
\hline $\begin{array}{c}\text { Moradia e Vida no } \\
\text { Lar }\end{array}$ & $87,5 \%$ & $82,2 \%$ & $80,0 \%$ & $82,9 \%$ & $82,7 \%$ \\
\hline Trabalho e Renda & $56,3 \%$ & $48,9 \%$ & $34,0 \%$ & $39,0 \%$ & $43,5 \%$ \\
\hline Lazer e Vida Social & $84,4 \%$ & $86,7 \%$ & $88,0 \%$ & $78,0 \%$ & $84,5 \%$ \\
\hline Percepção de Saúde & $65,6 \%$ & $88,9 \%$ & $76,0 \%$ & $78,0 \%$ & $78,0 \%$ \\
\hline $\begin{array}{c}\text { Segurança e Acessibi- } \\
\text { lidade }\end{array}$ & $37,5 \%$ & $44,4 \%$ & $40,0 \%$ & $39,0 \%$ & $40,5 \%$ \\
\hline
\end{tabular}

${ }^{1}$ Pessoas com escores 4 ou 5 na escala de cinco pontos descrita na seção de Métodos.

\section{Estilo de vida}

Os adultos com deficiência visual residentes na Grande Florianópolis apresentaram escore médio de $3,45(\mathrm{DP}=1,09)$, com mínimo de 0 e máximo de 5 pontos, para o estilo de vida.

As pessoas cegas, do sexo feminino, foram as que apresentaram a maior prevalência de respostas positivas na dimensão estilo de vida. Aproximadamente $51 \%$ dos adultos com deficiência visual apresentaram resultados positivos para o estilo de vida. A Tabela 3 apresenta os dados percentuais dos escores positivos em cada fator componente da dimensão estilo de vida.

Tabela 3. Percentuais de percepção positiva do estilo de vida ${ }^{1}$ - Adultos com deficiência visual da Grande Florianópolis, por grau de deficiência e gênero.

\begin{tabular}{cccccc}
\hline \multirow{2}{*}{ Componentes } & \multicolumn{2}{c}{ Cego } & \multicolumn{2}{c}{ Baixa Visão } & \multirow{2}{*}{ Geral } \\
\cline { 2 - 4 } & Mulheres & Homens & Mulheres & Homens & \\
\hline Relacionamentos & $87,5 \%$ & $84,4 \%$ & $78,0 \%$ & $80,5 \%$ & $82 \%$ \\
Controle do Estresse & $75,0 \%$ & $75,6 \%$ & $78,0 \%$ & $73,2 \%$ & $75 \%$ \\
Comportamento Pre- & $96,9 \%$ & $88,9 \%$ & $94,0 \%$ & $97,6 \%$ & $94 \%$ \\
ventivo & $40,6 \%$ & $37,8 \%$ & $40,0 \%$ & $43,9 \%$ & $40 \%$ \\
Atividade Física & $65,6 \%$ & $37,8 \%$ & $64,0 \%$ & $46,3 \%$ & $53 \%$ \\
Alimentação & &
\end{tabular}

${ }^{1}$ Pessoas com escores 4 ou 5 na escala de cinco pontos descrita na seção de Métodos.

Índice de qualidade de vida dos adultos com deficiência visual da Grande Florianópolis.

O índice de qualidade de vida dos adultos com deficiência visual (IQV-DV) foi determinado numa escala de zero a 10 pontos, a partir da soma dos escores nas duas dimensões do modelo de Nahas (2010): condições de vida e estilo de vida, cujos resultados 
foram apresentados anteriormente neste artigo. A análise categórica identificou o percentual de sujeitos com escore igual ou superior a oito pontos como positivos, entre quatro e oito pontos como intermediários, e menor ou igual a quatro como negativos.

Considerando essa categorização em três níveis, a prevalência de percepção positiva de qualidade de vida dos adultos com deficiência visual da Grande Florianópolis (percentual de indivíduos com escore igual ou maior que oito) foi de $33,9 \%$. Como pode ser visto na Figura 3, observou-se uma proporção maior de mulheres com escores positivos em comparação aos homens (36,6\% e 31,4\%, respectivamente).

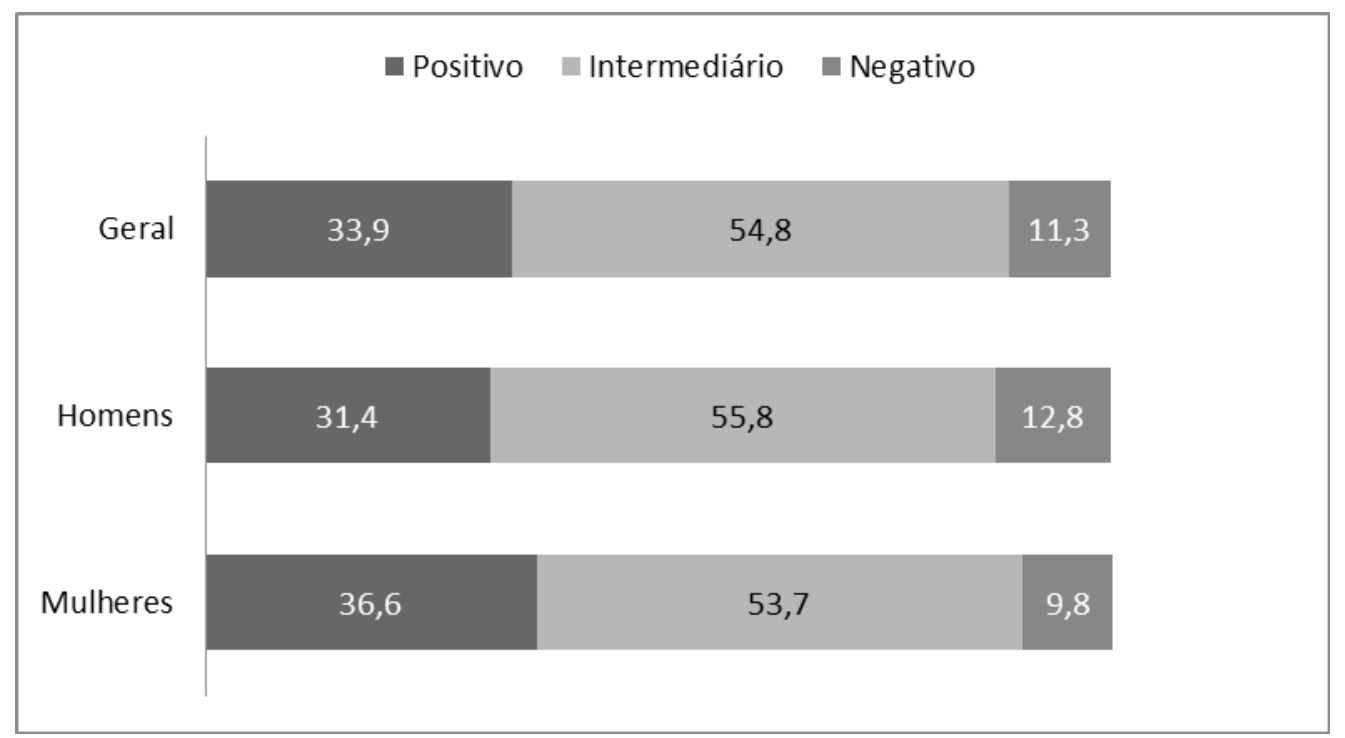

Figura 3. Índice de qualidade de vida dos adultos com deficiência visual da Grande Florianópolis, geral e por gênero.

$\mathrm{Na}$ Tabela 4, a percepção de qualidade de vida está mais bem detalhada, de acordo com as variáveis sociodemográficas. As variáveis idade e trabalho apresentaram-se associadas à percepção de qualidade de vida.

Tabela 4. Índice de qualidade de vida (IQV-DV) ${ }^{1}$, de acordo com as variáveis sociodemográficas, dos adultos com deficiência visual da Grande Florianópolis.

\begin{tabular}{lcccc}
\hline \multirow{2}{*}{ Variável } & \multirow{n}{*}{} & \multicolumn{3}{c}{ Índice de Qualidade de Vida } \\
\cline { 3 - 5 } & & Negativo & Intermediário & Positivo \\
\hline Gênero & 86 & $12,8 \%$ & $55,8 \%$ & $31,4 \%$ \\
Masculino & 82 & $9,8 \%$ & $53,7 \%$ & $36,6 \%$ \\
$\begin{array}{l}\text { Feminino } \\
\text { Idade }\end{array}$ & & & & \\
Adultos Jovens & 96 & $8,3 \%$ & $52,1 \%$ & $39,6 \%$ \\
Meia Idade & 72 & $15,3 \%$ & $58,3 \%$ & $26,4 \%$ \\
Escolaridade & & & &
\end{tabular}

Pensar a Prática, Goiânia, v. 17, n. 4, out./dez. 2014 


\begin{tabular}{|c|c|c|c|c|}
\hline$\leq 8$ anos & 119 & $14,3 \%$ & $53,8 \%$ & $31,9 \%$ \\
\hline$\geq 9$ anos & 49 & $4,1 \%$ & $57,1 \%$ & $38,8 \%$ \\
\hline \multicolumn{5}{|l|}{ Estado Civil } \\
\hline Com companheiro & 76 & $11,8 \%$ & $55,3 \%$ & $32,9 \%$ \\
\hline Sem companheiro & 92 & $10,9 \%$ & $54,3 \%$ & $34,8 \%$ \\
\hline \multicolumn{5}{|l|}{ Resquício Visual } \\
\hline Baixa Visão & 91 & $9,9 \%$ & $60,4 \%$ & $29,7 \%$ \\
\hline Cego & 77 & $13,0 \%$ & $48,1 \%$ & $39,0 \%$ \\
\hline \multicolumn{5}{|l|}{ Período de Incidência } \\
\hline Congênito & 107 & $11,2 \%$ & $50,5 \%$ & $38,3 \%$ \\
\hline Infância e Adolescência & 23 & $0,0 \%$ & $69,6 \%$ & $30,4 \%$ \\
\hline Adulto & 38 & $18,4 \%$ & $57,9 \%$ & $23,7 \%$ \\
\hline \multicolumn{5}{|l|}{ IMC } \\
\hline Faixa Normal & 71 & $8,5 \%$ & $56,3 \%$ & $35,2 \%$ \\
\hline Excesso de Peso & 97 & $13,4 \%$ & $53,6 \%$ & $33,0 \%$ \\
\hline \multicolumn{5}{|l|}{ Com quem mora } \\
\hline Sozinho & 17 & $23,5 \%$ & $52,9 \%$ & $23,5 \%$ \\
\hline Familiares & 136 & $8,8 \%$ & $55,1 \%$ & $36,0 \%$ \\
\hline Outras pessoas & 15 & $20,0 \%$ & $53,3 \%$ & $26,7 \%$ \\
\hline \multicolumn{5}{|l|}{ Trabalha $^{2}$} \\
\hline Sim & 91 & $6,6 \%$ & $46,2 \%$ & $47,3 \%$ \\
\hline Não & 77 & $16,9 \%$ & $64,9 \%$ & $18,2 \%$ \\
\hline TOTAL & & $11,3 \%$ & $54,8 \%$ & $33,9 \%$ \\
\hline
\end{tabular}

\section{Discussão}

Atualmente, mais da metade da população mundial vive em cidades. Estima-se que essa proporção chegará a $70 \%$ em 2050. Essa urbanização sem precedentes, com seus desdobramentos físicos, sociais e econômicos, tem um impacto na saúde e na qualidade de vida das pessoas, principalmente nos países em desenvolvimento.

Como todos os seres humanos, as pessoas com deficiência têm o direito de viver bem nas comunidades urbanas e fazer suas escolhas com segurança. Assim, fala-se muito em inclusão das pessoas com deficiência, para que possam desfrutar de uma vida com mais qualidade. A inclusão significa que a sociedade precisa adequar-se às características de cada pessoa com deficiência e não às características gerais (SASSAKI, 2008). Dessa forma, sentir-se incluído na sociedade deve significar que toda pessoa tem a capacidade e o direito de tomar parte ativa na vida da comunidade, cada qual à sua maneira e de acordo com seus interesses e possibilidades.

McDonnall (2007) destacou que aqueles adultos com deficiência visual que estão agregados a grupos e relacionamentos gerais possuem melhores condições de enfrentar desafios para manter um estilo de vida saudável. Gold, Shaw e Wolffe (2005) constataram, em seu estudo, que aproximadamente $56 \%$ das pessoas com baixa visão e $40 \%$ dos 
cegos, em uma pesquisa com 330 indivíduos, já encontraram desafios sociais em suas vidas.

Entretanto, não são todos que se sentem seguros em sair para as ruas. Montarzino et al. (2007) constataram em seu estudo que aproximadamente $62 \%$ sentem-se inseguros para caminhar à noite, e aproximadamente 50\% sentem-se inseguros em caminhar em locais desconhecidos, corroborando com os achados da pesquisa, na qual apenas $40,5 \%$ dos adultos com deficiência visual da Grande Florianópolis apresentaram percepção positiva no componente segurança e acessibilidade.

Quando verificado o trabalho e o resquício visual remanescente, aproximadamente $62 \%$ dos adultos cegos são trabalhadores, enquanto apenas $47 \%$ daqueles com baixa visão encontram-se no mercado de trabalho. Esses dados são surpreendentes, visto que aqueles adultos com um resquício visual apresentam maior possibilidade para utilizar essa visão remanescente, para estar se aperfeiçoando e adentrando no mercado de trabalho. Além disso, os achados contrapõem-se com o estudo de Gold, Shaw e Wolffe (2005), em pesquisa com jovens adultos (15 a 30 anos). Eles encontraram uma maior prevalência de trabalhadores com baixa visão, confirmando a literatura existente.

Fonseca (2008) destaca que, em razão de barreiras físicas e atitudinais, não se asseguram nem se alcançam os direitos humanos inerentes às liberdades de escolha e dignidade humana. Apesar de ainda haver barreiras no mercado de trabalho, verifica-se uma abertura maior em áreas em que as pessoas com deficiência visual podem não só exercer suas funções, mas, em muitos casos, exercê-las com melhor eficácia e credibilidade. Atividades como massoterapia ou assistência em câmaras de raios-X são alguns exemplos.

Essas atividades foram encontradas também no estudo de Gold, Shaw e Wolffe (2005), com predomínio em setores administrativos, serviços sociais ou educacionais. A reduzida opção de trabalho, segundo os autores, pode ser decorrente de barreiras, tais como: acesso restrito a materiais adaptados, equipamentos e informações; atitudes negativas de empregadores; consciência pública; melhor acesso ao transporte público; problemas pessoais; exigências do trabalho.

A Constituição Brasileira de 1988, em seu Capítulo II (Dos Direitos Sociais), art. 7o, inciso XXX, próíbe qualquer discriminação no que tange a salário ou critérios de admissão no trabalho, para as pessoas com deficiência. Fonseca (2008) enfatizou que, apesar de o Brasil estar caminhando positivamente, é fundamental que se supere a flagrante fragilidade que se deve a alguns problemas, como o próprio Beneficio de Prestação Continuada (BPC), o qual desestimula o emprego em prol de uma política assistencialista exagerada. As condições de trabalho e renda constituem um aspecto delicado e complexo, visto que abrangem não apenas questões de acessibilidade e oportunidade, mas também leis que já estão em vigor. Em muitos casos, tais leis favorecem o beneficiário em detrimento do mercado de trabalho, oferecendo benefícios e aposentadoria para adultos em condições de estar se preparando e adentrando nesse mercado.

Locais públicos ou privados, comerciais ou não, entretanto, devem buscar fazer as adaptações necessárias para atender todos os segmentos da população. Pequenas modificações podem proporcionar autonomia para essas pessoas viverem de forma mais independente nas atividades do dia a dia, tais como: trabalhar, fazer compras, atravessar ruas, caminhar com segurança nas ruas, praticar atividade física, entre outras.

Pensar a Prática, Goiânia, v. 17, n. 4, out./dez. 2014 
Certas instalações, como academias ou clubes, podem ser alternativas para a falta de acessibilidade arquitetônica nos ambientes ao ar livre, para indivíduos com defíciência visual ou mobilidade reduzida que queiram praticar atividade física regular. Rimmer et al. (2005) destacam, em seu estudo, que todas as 35 instalações (academias) pesquisadas nos EUA tinham um nível baixo ou moderado de acessibilidade, nos aspectos: ambiente construído, equipamentos, piscinas de natação, informações, políticas de instalação e comportamento profissional. Esses dados remetem a reflexões acerca das possibilidades nos países em desenvolvimento, visto que a pesquisa foi realizada em um país considerado desenvolvido.

Kirchner, Gerber e Smith (2008) encontraram resultados semelhantes sobre as principais barreiras enfrentadas no dia a dia. Com exceção da remoção de neve, que não há na região pesquisada neste estudo, as demais dificuldades giram em torno de problemas nas próprias ruas, em que a modificação de seu design contribuiria de forma significativa e removeria vários obstáculos diários. Montarzino et al. (2007) enfatizaram que a mobilidade desempenha um papel fundamental na qualidade de vida das pessoas com deficiência visual, assim como a falta de mobilidade pode acarretar a perda de emprego, redução de atividades recreativas e perda da autossuficiência.

Muitas pessoas com deficiência visual exibem uma marcha e postura padrão, como resultado de um ambiente inseguro sob a superproteção de seus pais e pouca participação em atividades físicas. Tais características, segundo os autores, são: cabeça para frente com cifose torácica, lordose lombar e marcha com pequena amplitude. Essa marcha é um indicativo de desenvolvimento motor pobre, acarretando uma grande barreira para o desenvolvimento motor, podendo influenciar no surgimento de algumas doenças e dores crônicas nesses adultos (CHEN; WANG, 2009).

Há evidências de que os adultos com deficiência visual possuem a saúde mais prejudicada, altas taxas de inatividade física e maiores níveis de sobrepeso e obesidade, havendo, dessa forma, necessidade de estratégias de promoção de saúde. Entre os adultos entrevistados na presente pesquisa, com relação à percepção de saúde, constatou-se que aproximadamente $67 \%$ possuem uma percepção positiva de sua saúde atual. Entretanto, quando analisados nesta pesquisa de modo geral, há alguns componentes fragilizados entre tal segmento da sociedade.

Esses componentes, McDonnall (2007) destacou em uma estrutura conceitual de atividades de promoção de saúde, que visem a melhorias em diversas áreas. Deve ser realizada intervenção direta de promoção de saúde, gerenciando a dieta e os níveis de atividade física dessa população, oportunizando áreas e atividades de lazer para uma participação mais ativa na sociedade.

A elevada taxa de inatividade física poderá gerar complicações para aqueles indivíduos sedentários, pois a inatividade física é associada a várias complicações médicas (NATIONAL INSTITUTES OF HEALTH, 2008).

Estudos do Department of Health and Human Services (2008) apresentaram evidências de que a falta de atividade física entre as pessoas com deficiência resulta em aumento do declínio funcional e uma maior ocorrência de condições secundárias. Essa instituição ainda explana que, para reduzir o risco de doenças crônicas, deve-se realizar pelo menos 30 minutos de atividades moderadas, na maioria dos dias da semana. Para manter o peso, a duração das atividades moderadas até vigorosas é em torno de 60 minutos, na 
maioria dos dias da semana. Contudo, para sustentar gradativamente a perda de peso, as atividades moderadas ou intensas devem ter duração de 60 a 90 minutos.

Quando os adultos com deficiência não são capazes de atender às orientações, devem engajar-se em atividades físicas regulares, de acordo com suas habilidades, evitando a inatividade física. Alguma atividade física é melhor do que nenhuma e todos os adultos devem evitar a inatividade (WHO, 2005).

Barreiras nos ambientes, assim como no próprio transporte público, ou falta de acesso aos locais de lazer podem dificultar a capacidade desses indivíduos de serem fisicamente ativos e manterem uma vida saudável. Apesar de as evidências existirem, que os adultos com deficiência visual são menos ativos fisicamente e possuem pior condição física que as pessoas que enxergam, pouco parece ter sido feito para mudar isso. A autora, em sua pesquisa, encontrou apenas dois estudos de intervenção com tal população, porém com uma amostra muito pequena (14 e 3), sendo o primeiro em um centro de reabilitação e o segundo com exercícios aeróbios para adultos totalmente cegos. Ambos os estudos constataram que os participantes possuem total condição de aprender e realizar as atividades, desde que dadas as oportunidades para o envolvimento (MCDONNALL, 2007).

Yeatts (2005) comparou pacientes com deficiência visual e um grupo controle de indivíduos saudáveis sem deficiência visual, mediante uma combinação de questionários com 105 questões. Ele constatou que os pacientes com deficiência visual apresentam uma redução na saúde física e mental, além da autoimagem. Lollar e Crews (2003) destacaram a importância de identificar e avaliar as condições secundárias provenientes de cada condição primária incapacitante. As limitações e doenças secundárias, quando evitadas, poderão, além de melhorar a qualidade de vida das pessoas, não gerar um impacto direto e negativo nos orçamentos de saúde em geral.

Nos últimos anos, várias pesquisas sobre intervenções relacionadas com a promoção da saúde foram relatadas, com relação à perda de peso e nível de atividade física para a população em geral (MCDONNALL, 2007). Porém, quando as restringimos ao grupo de pessoas com deficiência visual e a adultos, praticamente não há pesquisas sobre intervenção e promoção de saúde.

Além da prevenção de doenças, McDonnall (2007) destacou que programas de promoção de saúde e um estilo de vida saudável farão com que tais adultos tenham maiores condições de se manter no mercado de trabalho, possibilitando exercer seus direitos como cidadãos. Apesar de haver poucas evidências com relação a essa área, Kirchner, Schmeidler e Todorov (1999) afirmaram que adultos com deficiência visual saudáveis possuem maiores probabilidades de estar empregados que seus pares com um estilo de vida não saudável.

A promoção da saúde é importantíssima para todos os envolvidos, visto que o aumento da atividade física e a diminuição do peso corporal estão associados com menos problemas de saúde e, consequentemente, com diminuição dos custos com a saúde e melhora na qualidade de vida da população. Lollar e Crews (2003) afirmaram que intervenções desse tipo podem evitar a incidência de condições secundárias e maiores habilidades funcionais, resultando em uma melhora na qualidade de vida.

Pensar a Prática, Goiânia, v. 17, n. 4, out./dez. 2014 


\section{Conclusão}

Apesar do recente foco na promoção da saúde para as pessoas com deficiência, os adultos com deficiência visual ainda estão carentes de intervenções, não recebendo atenção adequada. Nesse campo de atuação, devem-se concentrar esforços para melhorar a saúde e o bem-estar dessa população.

Com relação aos fatores pessoais, essas intervenções podem ter um impacto positivo nas condições de saúde, nos níveis de atividade física, nos hábitos de vida, na autoeficácia e relacionados à qualidade de vida. Nos fatores ambientais, as intervenções devem se concentrar na facilitação de transporte público para todos os envolvidos.

Uma estratégia de promoção de saúde, por parte do poder público, poderia se configurar em orientações e assistência para a perda de peso, para que as pessoas se tornem mais ativas fisicamente e mantenham um estilo de vida saudável. Tal cenário poderia ser construído por intermédio de órgãos governamentais, em parceria com entidades destinadas a atender essa população.

A exposição dessa população em um processo de intervenção irá ajudar a prevenir a inatividade e reduzir o ganho de peso, a que, muitas vezes, a deficiência visual está as sociada. A essência da intervenção é criar um hábito saudável de vida e sua importância para a prevenção de doenças crônicas, principalmente. A intervenção propiciaria informações, experiências e habilidades essenciais para manter um estilo de vida saudável.

Observa-se que a percepção de qualidade de vida apresentou uma tendência nesta pesquisa quando analisado sob o período de incidência da deficiência visual. Quanto mais tarde as pessoas adquiriram a deficiência visual, menor é sua percepção positiva com relação à qualidade de sua vida. Isso pode ser decorrente de esses adultos ainda não se aceitarem como sujeitos com a deficiência visual.

As mulheres jovens, sem companheiros, com nove anos ou mais de estudo, que trabalham, foram as que apresentaram melhor percepção de qualidade de vida entre os adultos com deficiência visual da Grande Florianópolis. Além disso, as mulheres cegas de forma congênita, e que moram com seus familiares - foram as que apresentaram melhor percepção positiva de qualidade de vida.

Homens de meia idade - com baixa escolaridade, que adquiriram a deficiência na idade adulta e não trabalham - apresentaram uma prevalência maior para uma percepção negativa em sua qualidade de vida.

Deve-se pensar em estratégias de promoção para saúde, com viés de melhorias na qualidade de vida dos adultos que apresentaram uma prevalência maior para a percepção negativa. Isso por meio da mudança de hábitos do estilo de vida, principalmente no que se refere à alimentação e à atividade física, proporcionando melhores condições de vida para uma inclusão mais efetiva na sociedade. Tais mudanças devem ser pautadas principalmente nos aspectos de acessibilidade.

Pensar a Prática, Goiânia, v. 17, n. 4, out./dez. 2014 


\title{
QUALITY OF LIFE IN ADULTS WITH VISUAL IMPAIRMENT LIVING IN FLORI- ANÓPOLIS, SC
}

\begin{abstract}
The aim of this study was to evaluate quality of life (q.1.) in adults with visual impairment (v.i.). We interviewed 168 people (18-59 years). For data collection a semi-structured interview was used. The perception of q.1. tended when analyzed under period the incidence of disability. The later the people acquired v.i., the lower their positive perception of q.l. Young women, without partners, with nine or more years of study, employed, showed the best perception of q.1. among adults with v.i. One should think about strategies for health promotion, with improvements in q.l. of adults who had a higher prevalence of the negative perception

Keywords: Quality of life. Visually impaired persons. Social conditions. Life style.
\end{abstract}

\section{LA CALIDAD DE VIDA DE LOS ADULTOS CON DISCAPACIDAD VISUAL EN FLORIANÓPOLIS, SC}

\section{Resumen}

El objetivo de este estudio fue evaluar la calidad de vida (c.v.) en adultos con deficiencia visual (d.v.). Entrevistamos a 168 personas (18 a 59 años). Para la recolección de datos se utilizó una entrevista semi-estructurada. El percepción de c.v. ha tendido cuando se analizava el periodo de incidencia de la discapacidad. Las personas que más tarde adquirieron d.v. tenían/tuvieran menor percepción positiva sobre la c.v. Las mujeres jóvenes, que no tienen pareja, con nueve o más años de estudio, que trabajaban, mostraron la mejor percepción de c.v. entre los adultos con d.v. Se debe pensar en estrategias para la promoción de la salud, con mejoras en c.v. de adultos que tenían una mayor prevalencia de la percepción negativa.

Palabras-claves: Calidad de vida. Personas con daño visual. Condiciones sociales. Estilo de vida.

\section{Referências}

BITTENCOURT, Z. Z. L. C.; HOEHNE, E. L. Qualidade de vida de deficientes visuais. Medicina, Ribeirão Preto, v. 39, n. 2, p. 260-264, 2006. Disponível em: $<$ http://www.fmrp.usp.br/revista/2006/vol39n2/ao qualidade vida deficientes visuais1.p df $>$. Acesso em: 26 set. 2010.

BROWN, R. I. (Org.). Quality of life of people with disabilities. London: Stanley Thornes Publishers, 1997.

CAMPOS, M. O.; NETO, J. F. R. Qualidade de vida: um instrumento para promoção de saúde. Revista Baiana de Saúde Pública, v. 32, n. 2, p. 232-240, 2008. Disponível em: $<$ http://br.monografias.com/trabalhos-pdf/qualidade-vida-instrumento-promocaosaude/qualidade-vida-instrumento-promocao-saude.pdf $>$. Acesso em: 22 fev. 2010. 
CARVALHO, J. O perfil do estilo de vida relacionado à saúde do portador de deficiência visual da Grande Florianópolis. 1999. 102 f. Dissertação (Mestrado em Educação Física) - Centro de Desportos, Universidade Federal de Santa Catarina, Florianópolis. 1999.

CHEN, S.; WANG, Y. T. The impact of different walking environment on the gait and posture pattern of individuals with visual impairment. Asian Journal of Exercise \& Sports Science, v. 6, n. 1, p. 1-8, 2009.

DANTAS, R. A. S.; SAWADA, N. O.; MALERBO, M. B. Pesquisas sobre qualidade de vida: revisão da produção científica das universidades públicas do Estado de São Paulo. Revista Latino-Americana de Enfermagem, v. 11, n. 4, p. 532-538, 2003. Disponível em: $\quad<$ http://www.scielo.br/scielo.php?script=sci arttext\&pid=S0104$11692003000400017>$. Acesso em: 23 fev. 2010.

DEPARTMENT OF HEALTH AND HUMAN SERVICES (US). Physical activity guidelines for Americans. Atlanta, EUA: U.S. Department of Health and Human Services, Centers for Disease Control and Prevention, National Center for Chronic Disease Prevention and Health Promotion, 2008.

FLECK, M. P. A. O instrumento de avaliação de qualidade de vida da Organização Mundial da Saúde. Ciência \& Saúde Coletiva, v. 5, n. 1, p. 33-38, 2000. Disponível em: $<$ http://www.scielo.br/scielo.php?pid=S1413-81232000000100004\&script=sci_arttext $>$. Acesso em: 22 fev. 2010.

FLECK, M. P. A.; LEADL, O. F.; LOUZADA, S.; XAVIER, M.; CHACHAMOVICH, E.; VIEIRA, G.; SANTOS, L.; PINZON, V. Desenvolvimento da versão em português do instrumento de avaliação de qualidade de vida da OMS (WHOQOL-100). Revista Brasileira de Psiquiatria, v. 21, $\quad$ n. 1, p. 19-28, 1999. Disponível em: $<$ http://www.scielo.br/scielo.php?pid=S1516$\underline{44461999000100006 \& \text { script }=\text { sci abstract\&tlng }=\mathrm{pt}>}$. Acesso em: 22 fev. 2010.

FONSECA, R. T. M. Artigo 27 - Trabalho e emprego. In: RESENDE, A. P. C.; VITAL, F. M. P. A Convenção sobre Direitos das Pessoas com Deficiência comentada. Brasília: Secretaria Especial dos Direitos Humanos, Coordenadoria Nacional para Integração da Pessoa Portadora de Deficiência, 2008. p. 92-94.

GILL, T. M.; FEINSTEIN, A. R. A critical appraisal of the quality of quality-of-life measurements. JAMA, v. 272, n. 8, p. 619-626, 1994.

GOLD, D.; SHAW, A.; WOLFFE, K. The status of Canadian youth who are blind or visually impaired: a study of lifestyles, quality of life and employment. International Congress Series, n. 1282, p. 1148-1152, 2005.

GOUVEIA, N. Saúde e meio ambiente nas cidades: os desafios da saúde ambiental. Saúde e Sociedade, v. 8, $\quad$ n. 1, p. 49-61, 1999. Disponível em: 
$<$ http://www.scielo.br/scielo.php?pid=S0104-12901999000100005\&script=sci_arttext $>$. Acesso em: 23 fev. 2011.

KIRCHNER, C. E.; GERBER, E. G.; SMITH, B. C. Community barriers to physical activity for people with visual or motor impairments. American Journal of Preventive Medicine, v. 34, n. 4, p. 349-352, 2008.

KIRCHNER, C. E.; SCHMEIDLER, E.; TODOROV, A. Looking at Employment through a Lifespan Telescope: Age, Health, and Employment Status of People with Serious Visual Impairment. Mississippi State University, 1999, 117p.

LOLLAR, D. J.; CREWS, J. E. Redefining the role of public health in disability. Annual Review of Public Health, v. 24, p. 195-208, 2003.

McDONNALL, M. C. The need for health promotion for adults who are visually impaired. Journal of Visual Impairment \& Blindness, v. 101, n. 3, p. 133-145, 2007.

MONTARZINO, A. et al. The impact of mobility and public transport on the independence of visually impaired people. Visual Impairment Research, v. 9, p. 67-82, 2007.

MOSQUERA, C. Educação física para deficientes visuais. Rio de Janeiro: Sprint, 2000. $104 \mathrm{p}$.

NAHAS, M. V. Atividade física, saúde e qualidade de vida: conceitos e sugestões para um estilo de vida ativo. 5. ed. rev. e atual. Londrina: Midiograf, 2010.

. Atividade física, saúde e qualidade de vida: conceitos e sugestões para um estilo de vida ativo. 6. ed. rev. e atual. Londrina: Midiograf, 2013.

NATIONAL INSTITUTE OF HEALTH. Clinical guidelines on the identification, evaluation, and treatment of overweight and obesity in adults: the evidence report. 1. ed. International Medical Publishing, 2008. 264 p.

RESENDE, M. C.; JÚNIOR, R. L. Artigo 23 - Respeito pelo Lar e pela Família. In: RESENDE, A. P. C.; VITAL, F. M. P. A Convenção sobre Direitos das Pessoas com Deficiência comentada. Brasília: Secretaria Especial dos Direitos Humanos, Coordenadoria Nacional para Integração da Pessoa Portadora de Deficiência, 2008. p. 80-83.

RIMMER, J. H. et al. Accessibility of health clubs for people with mobility disabilities and visual impairments. American Journal of Public Health, v. 95, n. 11, p. 2022-2028, 2005.

SASSAKI, R. K. Artigo 24 - Educação. In: RESENDE, A. P. C.; VITAL, F. M. P. A Convenção sobre Direitos das Pessoas com Deficiência comentada. Brasília: Secretaria Especial dos Direitos Humanos, Coordenadoria Nacional para Integração da Pessoa Portadora de Deficiência, 2008. p. 83-85.

Pensar a Prática, Goiânia, v. 17, n. 4, out./dez. 2014 
SCHERER, R. L. Qualidade de vida de adultos com deficiência visual da grande Florianópolis. 2012. 138 p. Dissertação (Mestrado) - Programa de Pós-Graduação em Educação Física, Universidade Federal de Santa Catarina, 2012. Disponível em: $<$ http://repositorio.ufsc.br/xmlui/bitstream/handle/123456789/99373/304286.pdf? sequence $=1 \&$ is Allowed $=\mathrm{y}>$. Acesso em: 15 nov. 2013 .

SCHNEIDER, H. J.; FRIEDRICH, N.; KLOTSCHE, J. et al. The predictive value of different measures of obesity for incident cardiovascular events and mortality. The Journal of Clinical Endocrinology \& Metabolism, Washington, D.C., v. 95, n. 4, p. 1777-1785, 2010.

SEIDL, E. M. F.; ZANNON, C. M. L. C. Qualidade de vida e saúde: aspectos conceituais e metodológicos. Caderno de Saúde Pública, Rio de Janeiro, v. 20, n. 5, p. 580-588, 2004. Disponível em: $\quad<$ http://www.scielo.br/scielo.php?script=sci arttext\&pid=S0102311X2004000200027>. Acesso em: 22 fev. 2010.

STELMACK, J. Quality of life of low-vision patients and outcomes of low-vision rehabilitation. Optometry Vision Science, v. 78, n. 5, p. 35-42, 2001. Disponível em: $<$ http://www.ncbi.nlm.nih.gov/pubmed/11384011>. Acesso em: 26 set. 2010.

WHO. International Statistical Classification of Diseases and Related Health Prob$<$ http://apps.who.int/classifications/apps/icd/icd10online2005/fr-icd.htm>. Acesso em: 16 ago. 2010.

WHOQOL Group. Versão em português dos instrumentos de avaliação de qualidade de vida (WHOQOL, 1998). Disponível em: <http://www.ufrgs.br/psiq/whoqol1.html>. Acesso em: 13 abr. 2011.

YEATTS, P. Quality of life in patients with graves ophthalmopathy. Transactions of the American Ophthalmologic Society, v. 103, p. 368-411, 2005.

Recebido em: 20/03/2014

Revisado em: 21/08/2014

Aprovado em: 21/09/2014

Endereço para correspondência:

rogerlscherer@gmail.com

Roger Lima Scherer

Universidade Federal de Santa Catarina - Campus Reitor João David Ferreira Lima

Florianópolis - Santa Catarina - Brasil

CEP: $88040-900$

Pensar a Prática, Goiânia, v. 17, n. 4, out./dez. 2014 